\title{
The Sentinel Node Procedure in Breast Cancer: Nuclear Medicine as the Starting Point
}

\author{
Elif Hindié ${ }^{1}$, David Groheux ${ }^{1}$, Isabelle Brenot-Rossi ${ }^{2}$, Domenico Rubello ${ }^{3}$, Jean-Luc Moretti ${ }^{\dagger 1}$, and Marc Espié ${ }^{4}$ \\ ${ }^{I}$ Nuclear Medicine, Saint-Louis Hospital, University of Paris VII, Paris, France; ${ }^{2}$ Nuclear Medicine, Institut Paoli Calmettes, \\ Marseille, France; ${ }^{3}$ Nuclear Medicine-PET Unit, S. Maria della Misericordia Hospital, Insituto Oncologico Veneto, Rovigo, Italy; \\ and ${ }^{4}$ Medical Oncology, Breast Diseases Unit, Saint-Louis Hospital, Paris, France
}

\begin{abstract}
Learning Objectives: On successful completion of this activity, participants should be able to describe (1) the factors influencing the false-negative rate of SNB in breast cancer; (2) the risks associated with the use of the technique in specific situations such as large tumors, multiple ipsilateral tumors, or the setting of neoadjuvant chemotherapy; and (3) the meaning of internal mammary drainage on lymphoscintigraphy in terms of risk of occult internal mammary involvement.

Financial Disclosure: The authors of this article have indicated no relevant relationships that could be perceived as a real or apparent conflict of interest. CME Credit: SNM is accredited by the Accreditation Council for Continuing Medical Education (ACCME) to sponsor continuing education for physicians. SNM designates each JNM continuing education article for a maximum of 1.0 AMA PRA Category 1 Credit. Physicians should claim only credit commensurate with the extent of their participation in the activity.

For CE credit, participants can access this activity through the SNM Web site (http://www.snm.org/ce_online) through March 2012.
\end{abstract}

Axillary node status is a major prognostic factor in early breast cancer. Staging with sentinel node biopsy (SNB) leads to a substantial reduction in surgical morbidity. Recent multiinstitutional studies revealed SNB false-negative rates ranging from $5.5 \%$ to $16.7 \%$, higher than the target $(<5 \%)$ set by the 2005 guidelines of the American Society of Clinical Oncology. These alarming data point to the necessity of optimization. Dual mapping with radiotracer and blue dye, combining 2 different injection sites, and routinely using lymphoscintigraphy may improve accuracy. Factors associated with decreased sensitivity, such as prior excisional biopsy or neoadjuvant chemotherapy, should be recognized. The use of SNB in situations with a high prevalence of node positivity (large tumor, multifocality) is controversial. The risk of missed disease after negative SNB ranges from $1 \%$ to $4 \%$ in patients with $\mathrm{T} 1$ tumor and up to $15 \%$ in patients with T3. With peritumoral injection, internal mammary drainage is seen in about $20 \%$ of cases. Patients combining internal mammary drainage with a positive axillary sentinel node have close to a $50 \%$ probability of internal mammary involvement. Lymphoscintigraphy might thus be helpful in selecting patients for whom internal mammary radiation has a high benefit-to-risk ratio.

Key Words: sentinel node biopsy; breast cancer; micrometastases; lymphoscintigraphy; internal mammary node; radiation therapy; neoadjuvant chemotherapy

J Nucl Med 2011; 52:405-414

DOI: $10.2967 /$ jnumed.110.081711

\footnotetext{
Received Jul. 27, 2010; revision accepted Dec. 8, 2010.

For correspondence or reprints contact: Elif Hindié, Service de Médecine Nucléaire, Hôpital Saint-Louis, Assistance Publique-Hôpitaux de Paris, 1 Avenue Claude Vellefaux, 75475 Paris Cedex 10, France.

E-mail: elif.hindie@sls.aphp.fr

tDeceased.

COPYRIGHT @ 2011 by the Society of Nuclear Medicine, Inc.
}

B reast cancer is the most common class of cancer diagnosed in women worldwide. Estimates for 2010 in the United States were 209,060 new cases of invasive breast cancer and 40,203 deaths (1). Axillary node status is a major prognostic factor in early-stage disease, and this information is important for tailoring of treatment $(2,3)$.

Because imaging techniques have limited sensitivity, the axilla must be explored surgically. Traditional staging requires levels I and II axillary lymph node dissection (ALND) with 10 or more removed nodes (3). Axillary involvement is found in $10 \%-30 \%$ of patients with $\mathrm{T} 1(\leq 2 \mathrm{~cm})$ tumors, depending on size. This rate reaches $45 \%$ for small T2 tumors $(2.1-3 \mathrm{~cm})$ and $55 \%-70 \%$ for larger tumors $(2,4)$. However, routine ALND carries the risk of lymphedema, sensory disturbances, and chronic pain.

Sentinel node biopsy (SNB) is a less invasive method of checking for nodal involvement (5). SNB is based on the assumption of an orderly progression of lymph node invasion by malignant cells from a tumor site. The implication is that the entire basin is free of involvement if the first relay node is uninvolved (6). Patients with metastasis to a sentinel node would undergo either immediate or delayed completion ALND.

On a size basis, a metastasis in a sentinel node is designated a macrometastasis when larger than $2 \mathrm{~mm}$, a micrometastasis ( $\mathrm{pN} 1 \mathrm{mi}$ ) when larger than $0.2 \mathrm{~mm}$ but no more than $2 \mathrm{~mm}$, and isolated tumor cell clusters ( $\mathrm{pNOi}+$ ) when $0.2 \mathrm{~mm}$ or smaller and having no more than 200 tumor cells in a single cross section. This terminology stands whatever the detection method, whether classic staining or immunohistochemistry (7). 
The SNB procedure uses a radiotracer, a blue dye, or both $(8,9)$. Radiopharmaceuticals for SNB are colloids labeled with ${ }^{99 \mathrm{~m} T c}(10-12)$. They enter the lymphatic system and are engulfed by histiomonocytic cells of the sentinel node. They allow sentinel node visualization with a $\gamma$-camera before surgery and intraoperative detection with a hand-held $\gamma$-ray probe. Individual sentinel nodes take up about $1 \%$ of the locally injected activity. Dyes bind weakly to interstitial proteins, mostly albumin, and cause the blue coloring as they pass slowly through the sentinel node. Despite a risk of allergic reactions to blue dye, most teams favor the dual-mapping procedure $(8,9)$.

Which women can be offered SNB is a highly debated issue. Some centers use SNB only in patients with a unifocal tumor smaller than $2-3 \mathrm{~cm}$, whereas others have extended the application to patients with large T2 or T3 $(>5$ $\mathrm{cm}$ ) tumors or multiple ipsilateral carcinomas (multifocal/ multicentric) or to patients who have received neoadjuvant chemotherapy.

Here, we present a critical analysis of SNB in breast cancer in light of recent data that have been published mostly in the last 5 years.

\section{SNB STRATEGY AND SURGICAL MORBIDITY}

Randomized trials in which the primary aim was assessment of morbidity conclusively demonstrated a marked diminution of complications associated with the SNB strategy, when compared with routine ALND $(13,14)$. In the ALMANAC trial, 1,031 patients were randomized to undergo either ALND or SNB. Twelve months after surgery, the risk of lymphedema was $13 \%$ in the ALND group and $5 \%$ in the SNB group. The risk of sensory loss was, respectively, $31 \%$ and $11 \%$. Overall, patient-recorded scores for quality of life and arm functioning were significantly better in the SNB group (14). No specific analysis was performed, however, to see whether the morbidity advantage also applies to the small subset of patients with T2 tumor, in whom the risk of completion ALND is higher.

\section{LOCOREGIONAL RECURRENCE AND SURVIVAL}

Few randomized trials have investigated the impact of SNB strategy on recurrence and survival (15-17). All these trials compared SNB to SNB + ALND but not SNB to ALND.

The single-institution trial from Milano included 516 patients with tumors no larger than $2 \mathrm{~cm}$. The mean followup was 95 mo (15). The axillary relapse rate among patients with a negative sentinel node in the SNB group was as low as $1.2 \%$ (2/167). Interestingly, a rate of $4.6 \%$ would have been expected from the findings for the group SNB + routine ALND. The authors suggest that many small metastases might stay dormant if devoid of cancer stem cells. Although these data are fully reassuring, the wide exclusion criteria adopted might limit the generalization of these conclusions. The authors excluded patients with tumors that were larger than $2 \mathrm{~cm}$ or multicentric, patients with prior excisional biopsy or younger than $40 \mathrm{y}$, and patients for whom a sentinel node was not found at lymphoscintigraphy or on preoperative probe-guided inspection. Patients were also excluded after surgery if final pathologic examination revealed a tumor that was larger than $2 \mathrm{~cm}$ or multifocal (15).

The Sentinella/GIVOM trial (Gruppo Interdisciplinare Veneto di Oncologia Mammaria) included 749 women with a palpable tumor no larger than $3 \mathrm{~cm}$. During a median follow-up of $55.6 \mathrm{mo}$, locoregional recurrence occurred in 16 patients in the SNB group versus 3 in the ALND group. The 5-y relapse-free survival rate was slightly but not significantly lower in the SNB group (87.6\% vs. $89.9 \%$ ). The number of enrolled patients was deemed not sufficient to draw conclusions (16).

The National Surgical Adjuvant Breast and Bowel Project trial B-32 (NSABP B-32) randomized 5,611 women to SNB versus SNB + ALND. The primary endpoint was comparing overall survival in patients with a negative sentinel node in both arms (17). Tumor size was $2 \mathrm{~cm}$ or less in $83.8 \%, 2.1-4 \mathrm{~cm}$ in $14.7 \%$, and more than $4 \mathrm{~cm}$ in $1.5 \%$ of sentinel node-negative patients. During a mean follow-up of $95.6 \mathrm{mo}, 169$ of 2,011 sentinel node-negative patients in the SNB group and 140 of 1,975 in the SNB + ALND group died (hazard ratio, 1.2 by log-rank comparison; 95\% confidence interval, $0.96-1.5 ; P=0.12$ ). The $8-y$ overall survival rate was $90.3 \%$ in the SNB arm versus $91.8 \%$ in the ALND arm. Follow-up is continuing for longer-term assessment (17).

In total, data from patients with unifocal $\mathrm{T} 1$ tumor are fully reassuring, but it still seems premature to draw conclusions on other situations. Specific data from patients with T2 and T3 tumors would clearly be helpful.

\section{ALARMING RESULTS CONCERNING FALSE-NEGATIVE RATE}

In 2005, guidelines from the American Society of Clinical Oncology (ASCO) stressed that a multidisciplinary team should aim at a sentinel node identification rate of $85 \%$ with a false-negative rate of $5 \%$ or less in order to abandon axillary dissection (8). Identification rate is the proportion of patients in whom at least 1 sentinel node is found at operation. False-negative rate is the proportion of axillary node dissection-positive cases with a negative sentinel node at biopsy. Some false-negatives may result from massive involvement of the first relay node, a circumstance that interferes with the uptake of both radiocolloid and dye and diverts lymph flow to a node other than the true sentinel node $(6)$. The false-negative rate is the safety parameter of the SNB procedure (8). However, once SNB has been adopted, the false-negative rate is no longer measurable in clinical practice.

A metaanalysis of 69 early trials with a total of 8,059 patients in whom SNB was systematically followed by axillary dissection showed substantial variability in SNB performance (18). A high identification rate was associated 
with a low false-negative rate (18). On the basis of this metaanalysis, ASCO experts stated that "The strongest predictor of the false-negative rate across trials appears to be the proportion of patients for whom mapping is successful." Identification rate may thus "serve as a reasonable quality indicator for the SNB procedure" (8).

However, recent results from large multiinstitutional trials $(16,19-21)$ showed that all have achieved excellent identification rates, ranging from $93.5 \%$ to $97.2 \%$, but that none achieved a false-negative rate lower than 5\% (Table 1). The false-negative rate was $9.8 \%$ in the NSABP B-32 trial (21) and was dramatically high (16.7\%) in the Sentinella/GIVOM (16). Identification rate may thus provide false reassurance about the quality of the SNB procedure.

The false-negative rates ranged from 5.5\% to $16.7 \%$, with a weighted average of $9.2 \%$. The lowest false-negative rates were obtained in the 2 studies in which preoperative lymphoscintigraphy and dual mapping during surgery were required (Table 1). Direct comparison of protocols between different studies is, however, not possible since they refer to different surgeons and different settings.

False-negative SNB results might impair patient outcome for several reasons: missed nodes might lead to axillary recurrence that is difficult to treat, tumoral axillary nodes are a potential source of distant metastases, and understaging affects decisions about systemic therapy and specific radiation therapy to the chest wall and nodal basins $(3,22)$.

\section{OPTIMIZATION OF SNB PROCEDURE}

\section{Impact of Prior Excisional Biopsy on \\ False-Negative Rate}

Surgical biopsy can change the pattern of lymphatic drainage. In the NSABP B-32 trial (21), previous excisional biopsy did not affect sentinel node identification but was, however, associated with almost a doubling of false-negative rate compared with percutaneous biopsy (15.3\% [27/177] vs. $8.1 \%$ [48/589]; $P=0.0082$ ).

\section{Importance of Dual Mapping}

In the Axillary Lymphatic Mapping Against Nodal Axillary Clearance (ALMANAC) validation study, the false-negative rate was $6.7 \%$. However, if only blue sentinel nodes are considered, the false-negative rate was $9.1 \%$. Likewise, the false-negative rate would have been $10.9 \%$ based on isotope alone (20). In the Sentinella/GIVOM trial (16), blue dye was not used and the false-negative rate was higher than in the other trials (Table 1).

Isosulfan blue is in greater use in the United States, and patent blue V, in Europe (23). Data from NSABP B-32 and American College of Surgeons Oncology Group trial Z0010 (ACOSOG-Z0010) with isosulfan, and from ALMANAC with patent blue $\mathrm{V}$, showed that the overall risk of allergic reaction is close to $1 \%$ for both dyes, with an approximately $0.1 \%$ risk of severe reactions (grade III).

Mariani et al. suggested that ${ }^{99 \mathrm{~m} T c-l a b e l e d ~ c o l l o i d s ~ w i t h ~}$ most of the particles in the 100- to 200-nm size range would 
be ideal for SNB in breast cancer (10). The choice of tracer is often guided by local availability (23). ${ }^{99 \mathrm{~m}}$ Tc-labeled colloids of human serum albumin are often used in Europe. Among sulfur preparations, ${ }^{99 \mathrm{~m}}$ Tc-sulfur colloid is used in the United States (sometimes after filtration through a 0.1or $0.2-\mu \mathrm{m}$ membrane); ${ }^{99 \mathrm{~m} T c-r h e n i u m ~ s u l f i d e ~ c o l l o i d, ~ i n ~}$ Europe; and ${ }^{99 \mathrm{~m}}$ Tc-antimony trisulfide, in Australia. Because the clearing capacity of the macrophages in the sentinel node is limited, a high specific activity can improve sentinel node detection (10). There is no established difference between a 1 -d protocol (same-day imaging and surgery) and a 2-d protocol. Injected activity needs to be adapted.

\section{Resection of All Hot or Blue Nodes}

Differentiating a true sentinel node from a secondary echelon node is difficult. Also, lymphatics of a tumor site can drain simultaneously to more than 1 sentinel node.

Both the NSABP-B32 trial and the ALMANAC validation study showed the influence of the number of resected nodes on the false-negative rate $(20,21)$. In NSABP B-32, the false-negative rates was $17.7 \%$ if only 1 node was resected, $10 \%$ if $2,6.9 \%$ if $3,5.5 \%$ if 4 , and $1 \%$ if 5 or more (21). These results should not translate into routine removal of multiple nodes for an optimal SNB procedure, as this may have a detrimental impact on morbidity. However, all identified hot or blue nodes should be resected. Careful palpation by the surgeon of the operative field is also required to identify any suggestive large, hard nonblue and nonradioactive nodes.

\section{Effect of a Different Injection Site for Radiotracer and Blue Dye on False-Negative Rate}

In most early SNB studies, the tracer was injected around the tumor (5). Because the tracer is injected near the same lymph vessels that drain the tumor, such peritumoral injection is considered the gold standard against which all other mapping techniques are tested. Many investigators have reported good results using injection into the breast skin over the tumor, or using a periareolar or subareolar injection (24,25). These approaches exploit the common embryologic origin of the mammary gland and overlying skin and the description by Sappey in the 19th century that breast lymphatics collect in a subareolar plexus before draining to the axilla.

The only clearly established advantage of peritumoral injection is its ability to also reveal extraaxillary drainage. On the other hand, superficial injection techniques entail a faster lymphatic drainage, yield more radioactive counts at the axillary sentinel nodes, and are independent of the palpable or nonpalpable nature of the tumor (25).

In the prospective multiinstitutional randomized French study FRANSENODE, sentinel node identification rate with the $\gamma$-probe was $96 \%$ after peritumoral injection and 98.2\% after periareolar injection $(P=0.16$; not statistically significant). When detection by $\gamma$-probe plus blue dye was considered, the sentinel node detection rate reached $99.1 \%$, with no difference between peritumoral injection of the 2 tracers and periareolar injections (25). Because ALND was not performed, the impact of injection technique on the false-negative rate is unknown.

Lymphatic drainage of the breast is not completely understood (26). Rather than defending a particular injection site, it might be helpful not to use the same injection site for the radiotracer and blue dye to further enhance the complementary information obtained by combining 2 tracers (9). Anan et al. used 2 dyes: blue dye into the subareolar area and green dye peritumorally. The false-negative rate was $4.9 \%$. However, the false-negative rate was twice higher (10\%) if only blue nodes or only green nodes were considered (27). Thus, it might be appropriate to use 2 different injection sites; for example, peritumoral for the radiotracer and subareolar for blue dye $(9,28)$. Peritumoral injection of the radiotracer can evidence extraaxillary drainage (9).

\section{Improvement of SNB Procedure Through Lymphoscintigraphy}

By providing the surgeon with a map of sentinel nodes, scintigraphy has the potential to both improve accuracy and reduce morbidity relative to $\gamma$-probing alone $(10-12,29-32)$.

To identify all sentinel nodes and avoid confusion with a stasis in a lymphatic vessel, images are acquired with an adequate delay after injection. This delay may vary with the radiopharmaceutical, injection site, and patient characteristics. Lymphatic drainage can be slower in old or overweight patients. With planar scintigraphy, combining 2 views may help prevent some sentinel nodes from being missed (Fig. 1).

Lymphoscintigraphy identifies atypical drainage patterns. Drainage to the internal mammary basin occurs in $20 \%$ of patients after peritumoral injection (8). Other unusually located sentinel nodes are also seen in a nonnegligible percentage of patients: intramammary (prepectoral) in $6 \%$, interpectoral (Rotter's node) in $2 \%$, and infraclavicular (axilla level III) in 3\% (30).

The advent of SPECT/CT reinforces the potential of preoperative scintigraphy (33-35). Low-dose CT is sufficient
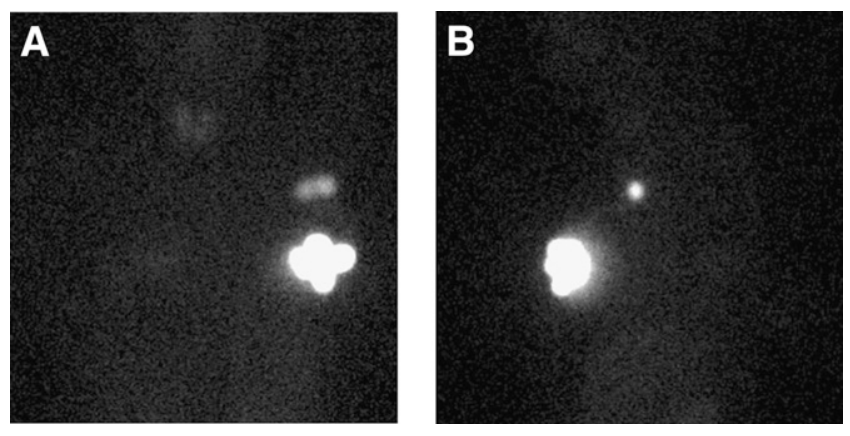

FIGURE 1. Lymphoscintigraphy of patient with nonpalpable left breast cancer $2 \mathrm{~h}$ after periareolar injection of ${ }^{99 \mathrm{mTc}} \mathrm{Tc}$-colloidal rhenium sulfide, with the 2 detectors positioned at right angles. (A) Anterior view shows 2 axillary sentinel nodes. (B) On left lateral view, only 1 axillary focus is seen; the 2 sentinel nodes are superimposed. 
B

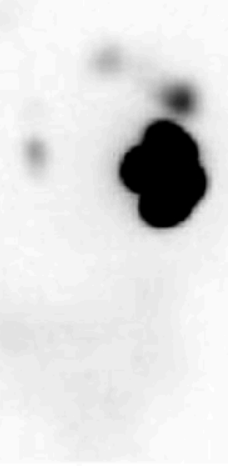

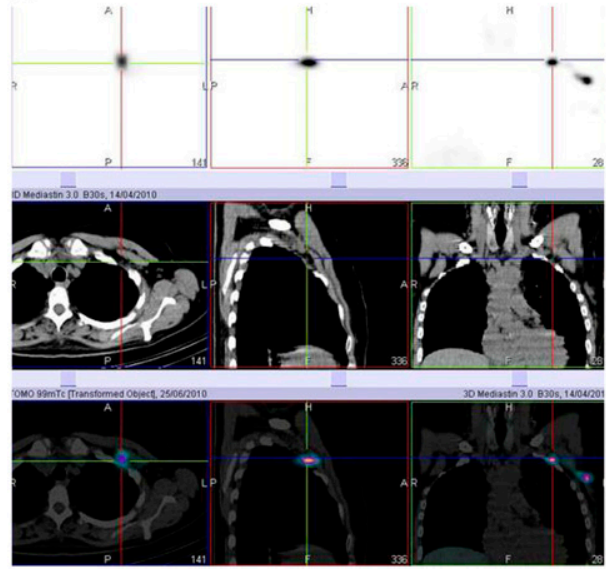

C

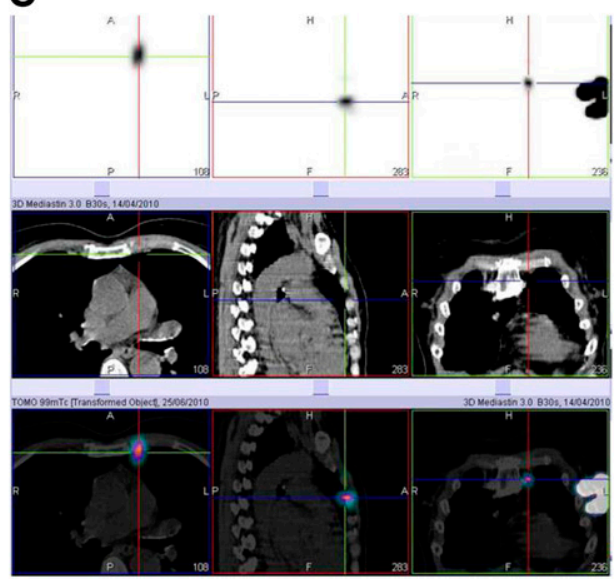

FIGURE 2. Lymphoscintigraphy of patient with 25-mm invasive lobular carcinoma of left breast. (A) Anterior planar view shows 2 axillary sentinel nodes plus internal mammary drainage. Medium-energy collimator was used to reduce star artifacts. (B) SPECT, CT, and SPECT/CT fusion images of axilla show upper sentinel node at Berg level II (beneath pectoralis minor) and other sentinel node at Berg level I. (C) Internal mammary node is in second intercostal space and did not undergo biopsy. Axillary sentinel nodes were negative.

to pinpoint atypically located sentinel nodes (Fig. 2). SPECT/CT can also detect hot nodes missed by planar imaging because of shine-through from the injection site or in overweight patients $(33,34)$. Thus, SPECT/CT can be useful when planar imaging is negative or ambiguous or shows unexpected drainage.

Failure to visualize a sentinel node is predictive of difficult intervention (31). Negative scintigraphy also heralds a higher risk of axillary involvement $(32,35)$. In a study by BrenotRossi et al, node invasion was found in $28.5 \%$ of patients with a visualized sentinel node versus $63.3 \%$ in cases of negative scintigraphy (32). In cases of nonvisualization after peritumoral injection, some authors reinject superficially. However, sentinel nodes that appeared with rescue injection were associated with a high (24\%) false-negative rate (36). Thus, when scintigraphy is negative after an adequate delay, one should check for the presence of macrometastases by ultrasound before surgery. When no sentinel node is identified at surgery, ALND should be performed $(3,8)$.

\section{SNB IN PATIENTS AT HIGH RISK OF NODAL INVOLVEMENT}

\section{SNB in Various Categories of Tumor Size}

In the ALMANAC validation study, the false-negative rate was $7.7 \%$ for patients with $\mathrm{T} 2$ tumors, not significantly different from that $(5.2 \%)$ for T1 tumors (20). Higher tumor grade was, however, associated with higher false-negative rate $(0 \%$ in grade $1,4.7 \%$ in grade 2 , and $9.6 \%$ in grade 3 tumors; $P=0.022)$. In the NSABP B-32 study $(21)$, the false-negative rate was $8.9 \%$ for patients with tumors $2.1-4$ $\mathrm{cm}$ and $10.3 \%$ for T1 tumors.

However, even assuming a constant false-negative rate, the negative predictive value decreases when the prevalence of lymph-node positivity in a population increases. A larger fraction of those patients with negative SNB results will harbor axillary disease.
Using technical data from the ALMANAC validation study (20) and from the group SNB + ALND of the NSABP B-32 trial (21), we assessed the risks of completion ALND and the risks of missed involvement. Our estimates based on ALMANAC data show that the need for completion ALND due to a positive sentinel node is almost twice higher for patients with T2 tumors $(2.1-5 \mathrm{~cm})$ than for patients with T1 tumors ( $51 \%$ vs. $28 \%$ ). The risk of missed axillary disease after a negative SNB is 4 times higher (9.3\% vs. $2.2 \%$ ) (Table 2 ). In the NSABP B-32, the tumor size category $(2.1-4 \mathrm{~cm})$ would correspond to small-to-medium T2 tumors. When the SNB procedure is applied to these patients, the need for completion ALND would be $41 \%$ (vs. $24 \%$ for T1); the risk of residual disease after negative SNB is also higher (6.2\% vs. $3.2 \%$ ) (Table 2).

There were too few patients with a T3 tumor in the ALMANAC, or a tumor larger than $4 \mathrm{~cm}$ in the NSABP B-32, to allow any conclusion to be drawn.

Taking into account node-positivity prevalence by tumor size category $(2,4)$ and assuming a false-negative rate of $10 \%$ (as found in the NSABP B-32), simple theoretic calculations would show that the risk of completion ALND increases progressively from $10 \%$ to $30 \%$ in patients with $\mathrm{T} 1$, to reach $60 \%$ in unselected patients with $\mathrm{T} 3$ tumor. Also, the risk of missed axillary disease after negative SNB would progressively increase from $1 \%$ to $4 \%$ in patients with T1 tumor, depending on tumor size, to about $15 \%$ in patients with $\mathrm{T} 3$ tumor.

\section{SNB in Patients with Multiple Simultaneous Ipsilateral Carcinomas}

In the ALMANAC validation study, the false-negative rate of SNB in 75 patients with multifocal tumors was $8.8 \%$ (20). In the prospective multiinstitutional French study IGASSU, with 211 evaluable patients in whom multifocal- 


\begin{tabular}{|c|c|c|c|c|c|c|c|}
\hline Trial & Category & $\begin{array}{c}\text { Patients } \\
\text { SNB + ALND }\end{array}$ & $\begin{array}{l}\text { Sentinel } \\
\text { node not } \\
\text { identified }\end{array}$ & $\begin{array}{c}\text { Positive } \\
\text { SNB cases }\end{array}$ & $\begin{array}{l}\text { Completion } \\
\text { ALND required }\end{array}$ & $\begin{array}{c}\text { SNB } \\
\text { false-negative } \\
\text { rate }\end{array}$ & $\begin{array}{l}\text { Estimates of } \\
\text { residual disease } \\
\text { after negative } \\
\text { sentinel node }\end{array}$ \\
\hline \multirow{2}{*}{$\begin{array}{l}\text { ALMANAC } \\
\text { validation } \\
\text { study }(20)\end{array}$} & $\mathrm{T} 1(\leq 2 \mathrm{~cm})$ & 458 & $3.5 \%(16)$ & $27.9 \%(128)$ & $31.4 \%(144)$ & $5.2 \%(7 / 135)$ & $2.2 \%(7 / 314)$ \\
\hline & T2 $(2.1-5 \mathrm{~cm})$ & 235 & $3.4 \%(8)$ & $51.1 \%(120)$ & $54.5 \%(128)$ & $7.7 \%(10 / 130)$ & $9.3 \%(10 / 107)$ \\
\hline \multirow{2}{*}{$\begin{array}{l}\text { NSABP } \\
\text { B32 }^{\dagger}(21)\end{array}$} & T1 & 2,201 & $3 \%^{\dagger}\left(65^{\dagger}\right)$ & $21.4 \%(472)$ & $24.4 \%\left(537^{\dagger}\right)$ & $10.3 \%(54 / 526)$ & $3.2 \%\left(54 / 1,664^{\dagger}\right)$ \\
\hline & $2.1-4 \mathrm{~cm}$ & 490 & $1.6 \%+\left(8^{\dagger}\right)$ & $39.6 \%(194)$ & $41.2 \%\left(202^{\dagger}\right)$ & $8.9 \%(19 / 213)$ & $6.6 \%\left(19 / 288^{\dagger}\right)$ \\
\hline $\begin{array}{l}\text { ALMANAC } \\
\text { validation } \\
\text { study (20) }\end{array}$ & Multifocal lesions & 75 & $5.3 \%(4)$ & $41.3 \%(31 / 75)$ & $46.7 \%(35 / 75)$ & $8.8 \%(3 / 34)$ & $7.5 \%(3 / 40)$ \\
\hline IGASSU (37) & Multicentric or multifocal & $211^{\ddagger}$ & $6.6 \%(14)$ & $42.2 \%(89)$ & $48.8 \%$ & $13.6 \%(14 / 103)$ & $12.9 \%(14 / 108)$ \\
\hline Neoadjuvant (40) & Neoadjuvant chemotherapy & 195 & $9.7 \%(19)$ & $23.6 \%(46)$ & $33.3 \%(65)$ & $11.5 \%(6 / 52)$ & $4.6 \%(6 / 130)$ \\
\hline
\end{tabular}

${ }^{*}$ Completion ALND is theoretically required when sentinel node is positive or not identified. Numbers are extracted from Tables 1 and 4 of ALMANAC (20). Data are based on patients with available information on tumor size. Only 12 patients had T3 tumor, not allowing comparison.

${ }^{\dagger}$ Numbers are extracted from Tables 1, 2, and 4 of NSABP B-32 (21). Rate of failed sentinel node identification is an approximation based on data from both arms combined.

FInjection was subareolar; $62 \%$ had dual mapping, and $37 \%$ had only radioisotope.

Data were extracted from ALMANAC validation phase (20), NSABP B-32 trial (group SNB + ALND) (21), IGASSU trial (Interest of Axillary Sentinel Lymph Node Biopsy in Multiple Invasive Breast Cancer) (37), and "Ganglion Sentinelle et Chimiothérapie Néoadjuvante" trial (40).

ity was diagnosed before SNB and breast surgery, the falsenegative rate was $13.6 \%$ (37).

Pooled data from these 2 studies show that the risk of completion ALND is close to 50\% and the estimated risk of missed axillary involvement after a negative SNB is $7.5 \%-$ $12.9 \%$ (Table 2).

\section{SNB After Neoadjuvant Chemotherapy}

Patients with tumors larger than $3 \mathrm{~cm}$ are often offered neoadjuvant chemotherapy to increase the probability of breast-conserving surgery. Performing SNB after neoadjuvant chemotherapy is a highly controversial topic $(3,8,38)$. Chemotherapy may increase the chance of false-negative findings due to lymphatic changes, fibrosis, and patchy killing $(6,8,38)$. SNB before neoadjuvant chemotherapy is more accurate (39) but means separate surgery and a possible delay in the start of chemotherapy.

A French study, "Ganglion Sentinelle et Chimiothérapie Néoadjuvante," prospectively assessed the accuracy of SNB after neoadjuvant chemotherapy (40). A sentinel node was identified in $90 \%$ of patients. The false-negative rate was $11.5 \%$. The data led to an estimate of missed axillary disease after negative SNB of $4.6 \%$ (Table 2). This risk cannot be neglected, as it occurs in patients who already received chemotherapy and might not receive adequate radiation because of false-negative SNB results.

The position of the National Comprehensive Cancer Network is to perform SNB before neoadjuvant chemotherapy and to base decisions about ALND and adjuvant treatment on the node status before chemotherapy (3).

\section{Safety Measures in Patients with High Probability of Axillary Involvement}

Ultrasound, coupled with fine-needle biopsy of suggestive nodes, can spare some patients with macrometastases an unnecessary SNB and the risk of false-negative results (41). Baruah et al. reported a sensitivity of $28.5 \%$ (39/137 node-positive patients were identified) and a specificity of $100 \%(41)$.

In a study by Heusner et al., the sensitivity of ${ }^{18}$ F-FDGPET/CT (with contrast-enhanced CT) was $58 \%$. The positive predictive value was $82 \%$ (42). It would be difficult to justify the use of this technique solely for patient selection before SNB. However, baseline ${ }^{18}$ F-FDG-PET/CT in patients with large tumors might influence management by identifying distant metastases or extraaxillary nodes (43). ${ }^{18} \mathrm{~F}-\mathrm{FDG}-\mathrm{PET} / \mathrm{CT}$ is also being investigated as a tool for early assessment of response to neoadjuvant chemotherapy (44).

Practices concerning intraoperative pathologic assessment of sentinel nodes vary $(14,16,19-21)$. However, intraoperative assessment would seem desirable when the prior probability of nodal involvement is high. Recall for completion ALND causes distress to patients, exposes them to the risks of a second anesthesia and operation, and increases total hospital stay. In the Sentinella/GIVOM trial, frozen-section examination offered a sensitivity of $75.3 \%$ (16). Imprint or touch-preparation cytology is less timeconsuming but requires experience in cytology reading. In the NSAPB B-32 trial, intraoperative cytology had $61.4 \%$ sensitivity and $99.5 \%$ specificity (21). 
Intraoperative molecular techniques have also been developed. One assay analyzes fresh homogenized tissue from sentinel nodes using reverse transcription-polymerase chain reaction to detect cytokeratin-19 and mammaglobin messenger RNAs and is calibrated to detect sentinel node metastases larger than $0.2 \mathrm{~mm}$ (45). In 1 study, sensitivity was $87.6 \%$, specificity $94.2 \%$, and positive predictive value $86.2 \%$ (45). Because specificity is still suboptimal, molecular tests might be better adapted to a population with a high probability of axillary involvement. Also, because alternate tissue slices analyzed with the assay are lost for postoperative histology, there is a potential risk of loss of information on small metastases.

\section{INTERNAL MAMMARY DRAINAGE: POTENTIAL IMPACT ON DECISIONS ABOUT RADIOTHERAPY}

In the last few years, there has been renewed interest in internal mammary treatment (46). A metaanalysis showed that postmastectomy radiotherapy to chest wall and nodal basins (including internal mammary) improves the survival of axilla-positive patients (22). Internal mammary radiation techniques have also improved, and cardiovascular side effects are less common $(22,46)$. However, internal mammary radiation remains controversial, mainly because of the difficulty of selecting patients at risk of occult internal mammary involvement (46). Difficulty in establishing guidelines was acknowledged by the National Comprehensive Cancer Network Panel (3). In some institutions, patients are selected on the basis of tumor location (medial/central), as is the case for the European Organisation for Research and Treatment of Cancer trial 22922 (EORTC 22922) assessing the impact of internal mammary radiation on survival.

Lymphoscintigraphy is an objective means of identifying cases of drainage to the internal mammary basin and thus with an anatomic substratum for occult internal mammary invasion.

\section{Probability of Internal Mammary Involvement in Patients with Internal Mammary Drainage According to Axilla Sentinel Node Status}

We reviewed studies in which internal mammary nodes identified on lymphoscintigraphy underwent biopsy. We selected 6 studies that fulfilled the following criteria: a large number of patients, peritumoral or intratumoral tracer injection, internal mammary biopsy successfully achieved in most patients with internal mammary drainage, and axilla staged routinely independent of internal mammary status (47-52).

These studies included a total of 3,876 patients (Table 3 ). Internal mammary drainage was present in $20.4 \%$. When internal mammary biopsy could be performed, it was positive in $17.2 \%$ of patients. A major difference was found according to axillary sentinel node status. In patients with a negative axilla, internal mammary biopsy was positive in $7.8 \%$, whereas in patients with positive axilla, internal mammary biopsy was positive in $41 \%$ (Table 3 ). Because it is difficult to perform multiple biopsies on patients with 
multiple internal mammary hot nodes, the true rate of invasion should be even higher, close to $50 \%$. This high risk of occult internal mammary involvement in cases of a positive axillary sentinel node and internal mammary drainage suggests that these patients can be offered internal mammary radiotherapy with no need for internal mammary biopsy.

\section{Patients with Internal Mammary Drainage and Negative Axillary Sentinel Node}

Internal mammary biopsy may identify a few candidates for internal mammary radiation (Table 3). However, internal mammary biopsy is linked to a small percentage of complications (due to the proximity of internal mammary vessels and pleura), which makes the procedure controversial.

In a patient with a small tumor, finding internal mammary invasion would also lead to chemotherapy. In the 2 studies that included only tumors smaller than $2-3 \mathrm{~cm}(48,52)$, internal mammary positivity in cases of internal mammary drainage and a negative axillary sentinel node was 5\% (Table 3).

\section{SNB AND SOME CONTROVERSIAL ISSUES}

\section{Prognostic Significance of Micrometastases and Isolated Tumor Cell Clusters}

The advent of SNB resulted in upstaging of approximately $10 \%$ of breast cancer patients, an increase mainly due to micrometastases (53).

Some retrospective studies found that patients with micrometastases $\mathrm{pN} 1 \mathrm{mi}$ or with isolated tumor cell clusters $\mathrm{pNO}(\mathrm{i}+)$ do not have worse disease-free survival than do sentinel node-negative patients (54). It was also found that completion ALND was associated with improved outcome in cases of sentinel node macrometastases $(>2 \mathrm{~mm})$ but not in cases of microscopic involvement (55). Bleiweiss suggested that some "tumor deposits" detected solely by anticytokeratin immunostaining could be benign epithelial cells transported to the sentinel node after breast biopsy (6).

Other retrospective studies found microscopic nodal disease that was clinically relevant (56-58). At completion ALND, Cox et al. found additional invasion in $15.5 \%$ of women with sentinel node micrometastasis. Survival was shorter in patients with micrometastases than in node-negative patients (56). Tan et al. reexamined paraffin blocks from 368 patients who underwent surgery long before and whose ALND was negative. The review procedure, with a technique similar to that used for sentinel nodes, revealed occult metastases in 83 patients $(23 \%)$. Death by cancer was significantly more frequent in these patients (57). De Boer used The Netherlands Cancer Registry to compare outcomes. After adjustment for other prognostic factors, the hazard ratio for disease events was 1.56 for patients with micrometastases and no systemic therapy, as compared with node-negative patients. The hazard ratio was 1.5 in cases of isolated tumor cell clusters (58). Outcome was improved with adjuvant therapy. For patients with micrometastases, 5-y disease-free survivals were $87.9 \%$ and $75.9 \%$, respectively, with and without systemic therapy $(P<0.001)(58)$. The prognostic relevance of microscopic nodal disease is thus an area of continuing controversy. Results of prospective trials (ACOSOG-Z0010, NSABP B-32, and International Breast Cancer Study Group trial 23-01 [IBCSG 23-01]) are eagerly awaited.

In the new edition of the AJCC Cancer Staging Manual (7), stage I breast cancer has been divided into stage IA and stage IB. Stage IB includes patients with a T1 tumor with only micrometastases (N1mi). These patients are thus excluded from stage IIA.

\section{Alternatives to Routine ALND in Patients with Positive Sentinel Node}

AMAROS is a trial of completion ALND versus axillary radiotherapy in patients with a positive sentinel node. IBCSG 23-01 is a trial of ALND versus no ALND for patients with sentinel node micrometastases. Until results are available, the current recommendations are to perform ALND in patients with a positive sentinel node (3).

The risk of additional node involvement depends on such factors as the number of positive and total sentinel nodes, size of sentinel node metastases, size of primary tumor, nuclear grade, and presence of lymphovascular invasion. Several models have thus been developed to help predict additional involvement (59). Some aim more specifically at patients with sentinel node micrometastases (60).

\section{SNB After Aesthetic Breast Surgery}

Although SNB is technically feasible in patients with a history of aesthetic breast surgery (61), the impact on falsenegative rate has not been studied.

\section{SNB and Pregnancy}

Blue dyes are contraindicated (8). Considering radiocolloids, the guidelines of ASCO did not validate their use in pregnancy (8). The European Association of Nuclear Medicine considered the isotopic technique safe, both in breast cancer and in melanoma, and the radiation dose potentially delivered to the fetus very low $(12,23)$. Gentilini et al. recommend using a low activity $(\leq 10 \mathrm{MBq})$, with imaging and surgery the same day (62).

\section{SNB in Ductal Carcinoma in Situ}

Core-needle biopsy may be affected by sampling error; in about $15 \%$ of patients with ductal carcinoma in situ, invasive disease is found at surgery (63). However, routine use of SNB in ductal carcinoma in situ is not recommended. SNB was positive in only $1.4 \%$ of unselected cases (64). SNB should be considered, however, when a mastectomy is planned, because SNB could not be performed afterward if invasive disease is diagnosed $(3,65)$. SNB can also be an option with breast-conserving surgery when there is a high risk of invasive cancer at final diagnosis (e.g., large clinical or mammographic mass or high nuclear grade).

\section{RESEARCH AREAS IN LYMPHATIC MAPPING}

\section{Axillary Reverse Mapping (ARM)}

A radiotracer is used to identify the breast sentinel node, and a blue tracer is injected in the inner part of the arm to 
visualize lymphatics and nodes draining the upper extremity and allow their preservation (66). Early reports have suggested that ARM lymph nodes do not contain metastatic disease and that the technique can reduce the incidence of lymphedema in cases of completion ALND (66). Other authors have used the technique during ALND in nodepositive patients and found a nonnegligible prevalence $(10 \%-20 \%)$ of disease involving ARM nodes (67). Thus, further research is needed to determine the appropriate population for this new procedure in order to preserve oncologic safety.

\section{SNB and Concomitant Probe-Guided Excision of Nonpalpable Breast Cancer}

Currently, nonpalpable breast lesions benefit from presurgical wire localization. ROLL (radioguided occult lesion localization) is a more patient-friendly technique that consists of injecting a radiotracer into the lesion under ultrasound or stereotactic guidance, followed by intraoperative $\gamma$-probing. In 1 study, complete tumor excision with tumor-free margins was achieved in $89.4 \%$ of patients undergoing ROLL versus $82.4 \%$ undergoing wire-guided lumpectomy (68).

The radiotracer can serve for concomitant SNB $(68,69)$. Van Rijk et al. applied this combined procedure (also called SNOLL) to 368 patients after intratumoral injection of 99m Tc-nanocolloid. Tumor-free margins were obtained in $89 \%$. The sentinel node could be identified in $97 \%$ of patients and was involved in 19\% (69). Thus, ROLL with concomitant SNB is a strategy that deserves further investigation.

\section{Intraoperative Imaging}

Intraoperative real-time imaging using a portable $\gamma$-camera is being investigated in situations with difficult-to-localize sentinel nodes (70). Its role in breast cancer remains to be clarified.

\section{CONCLUSION}

In a woman with a unifocal T1 tumor, most data suggest that the SNB procedure offers safe (and possibly improved) staging with reduced morbidity. This is the case for most newly diagnosed breast cancer patients. In patients with large $(>3 \mathrm{~cm})$ or multiple ipsilateral tumors, SNB is still debated. More effort should be spent on improving patient selection (through such techniques as ultrasonography with fine-needle biopsy or ${ }^{19} \mathrm{~F}-\mathrm{FDG}$ PET/CT) before SNB in high-risk patients.

Some recent multiinstitutional trials revealed high falsenegative rates. Reducing the false-negative rate of SNB is an important and difficult challenge. We highlighted some causes of failure and discussed several points that should help in optimizing the procedure.

Documentation of internal mammary drainage on lymphoscintigraphy might be helpful in the choice of locoregional treatment. If this information is to be fully appreciated, the peritumoral injection technique needs to be better standardized.
SNB is a team procedure. Interaction between nuclear physicians and clinicians is more necessary than ever.

\section{ACKNOWLEDGMENT}

Prof. Jean-Luc Moretti, a member of the Editorial Board of $J N M$, died on December 21, 2010, in Paris at the age of 64, after a long illness. His death is a painful loss to his family and friends and to the international community of nuclear medicine.

\section{REFERENCES}

1. Jemal A, Siegel R, Xu J, Ward E. Cancer statistics, 2010. CA Cancer J Clin. 2010;60:277-300.

2. Carter CL, Allen C, Henson DE. Relation of tumor size, lymph node status, and survival in 24,740 breast cancer cases. Cancer. 1989;63:181-187.

3. Carlson RW, Allred DC, Anderson BO, et al. Breast cancer: clinical practice guidelines in oncology. J Natl Compr Canc Netw. 2009;7:122-192.

4. Blamey RW, Hornmark-Stenstam B, Ball G, et al. ONCOPOOL: a European database for 16,944 cases of breast cancer. Eur J Cancer. 2010;46:56-71.

5. Krag DN, Weaver DL, Alex JC, Fairbank JT. Surgical resection and radiolocalization of the sentinel lymph node in breast cancer using a gamma probe. Surg Oncol. 1993;2:335-339.

6. Bleiweiss IJ. Sentinel lymph nodes in breast cancer after 10 years: rethinking basic principles. Lancet Oncol. 2006;7:686-692.

7. Edge SB, Byrd DR, Compton CC, Fritz AG, Greene FL, Trotti A. AJCC Cancer Staging Manual. 7th ed. New York, NY: Springer; 2010.

8. Lyman GH, Giuliano AE, Somerfield MR, et al. American Society of Clinical Oncology guideline recommendations for sentinel lymph node biopsy in earlystage breast cancer. J Clin Oncol. 2005;23:7703-7720.

9. Hindié E, Groheux D, Espie M, et al. Sentinel node biopsy in breast cancer. Bull Cancer. 2009;96:713-725.

10. Mariani G, Moresco L, Viale G, et al. Radioguided sentinel lymph node biopsy in breast cancer surgery. J Nucl Med. 2001;42:1198-1215.

11. Aarsvold JN, Alazraki NP. Update on detection of sentinel lymph nodes in patients with breast cancer. Semin Nucl Med. 2005;35:116-128.

12. Buscombe J, Paganelli G, Burak ZE, et al. Sentinel node in breast cancer procedural guidelines. Eur J Nucl Med Mol Imaging. 2007;34:2154-2159.

13. Purushotham AD, Upponi S, Klevesath MB, et al. Morbidity after sentinel lymph node biopsy in primary breast cancer: results from a randomized controlled trial. J Clin Oncol. 2005;23:4312-4321.

14. Mansel RE, Fallowfield L, Kissin M, et al. Randomized multicenter trial of sentinel node biopsy versus standard axillary treatment in operable breast cancer: the ALMANAC Trial. J Natl Cancer Inst. 2006;98:599-609.

15. Veronesi U, Viale G, Paganelli G, et al. Sentinel lymph node biopsy in breast cancer: ten-year results of a randomized controlled study. Ann Surg. 2010;251: 595-600.

16. Zavagno G, De Salvo GL, Scalco G, et al. A Randomized clinical trial on sentinel lymph node biopsy versus axillary lymph node dissection in breast cancer: results of the Sentinella/GIVOM trial. Ann Surg. 2008;247:207-213.

17. Krag DN, Anderson SJ, Julian TB, et al. Sentinel-lymph-node resection compared with conventional axillary-lymph-node dissection in clinically node-negative patients with breast cancer: overall survival findings from the NSABP B-32 randomised phase 3 trial. Lancet Oncol. 2010;11:927-933.

18. Kim T, Giuliano AE, Lyman GH. Lymphatic mapping and sentinel lymph node biopsy in early-stage breast carcinoma: a metaanalysis. Cancer. 2006;106:4-16.

19. Gill G; SNAC Trial Group of the Royal Australasian College of Surgeons (RACS) and NHMRC Clinical Trials Centre. Sentinel-lymph-node-based management or routine axillary clearance? One-year outcomes of sentinel node biopsy versus axillary clearance (SNAC): a randomized controlled surgical trial. Ann Surg Oncol. 2009;16:266-275.

20. Goyal A, Newcombe RG, Chhabra A, Mansel RE; ALMANAC Trialists Group. Factors affecting failed localisation and false-negative rates of sentinel node biopsy in breast cancer: results of the ALMANAC validation phase. Breast Cancer Res Treat. 2006;99:203-208.

21. Krag DN, Anderson SJ, Julian TB, et al. Technical outcomes of sentinel-lymphnode resection and conventional axillary-lymph-node dissection in patients with clinically node-negative breast cancer: results from the NSABP B-32 randomised phase III trial. Lancet Oncol. 2007;8:881-888.

22. Clarke M, Collins R, Darby S, et al. Effects of radiotherapy and of differences in the extent of surgery for early breast cancer on local recurrence and 15- 
year survival: an overview of the randomised trials. Lancet. 2005;366:20872106.

23. Chakera AH, Hesse B, Burak Z, et al. EANM-EORTC general recommendations for sentinel node diagnostics in melanoma. Eur J Nucl Med Mol Imaging. 2009;36:1713-1742.

24. McMasters KM, Wong SL, Martin RC II, et al. Dermal injection of radioactive colloid is superior to peritumoral injection for breast cancer sentinel lymph node biopsy: results of a multiinstitutional study. Ann Surg. 2001;233:676-687.

25. Rodier JF, Velten M, Wilt M, et al. Prospective multicentric randomized study comparing periareolar and peritumoral injection of radiotracer and blue dye for the detection of sentinel lymph node in breast sparing procedures: FRANSENODE trial. J Clin Oncol. 2007;25:3664-3669.

26. Suami H, Pan WR, Mann GB, Taylor GI. The lymphatic anatomy of the breast and its implications for sentinel lymph node biopsy: a human cadaver study. Ann Surg Oncol. 2008;15:863-871.

27. Anan K, Mitsuyama S, Kuga H, et al. Double mapping with subareolar blue dye and peritumoral green dye injections decreases the false-negative rate of dyeonly sentinel node biopsy for early breast cancer: 2 -site injection is more accurate than 1-site injection. Surgery. 2006;139:624-629.

28. Noguchi M, Inokuchi M, Zen Y. Complement of peritumoral and subareolar injection in breast cancer sentinel lymph node biopsy. J Surg Oncol. 2009; 100:100-105.

29. Kim SC, Kim DW, Moadel RM, et al. Using the intraoperative hand held probe without lymphoscintigraphy or using only dye correlates with higher sensory morbidity following sentinel lymph node biopsy in breast cancer: a review of the literature. World J Surg Oncol. 2005;3:64.

30. van Rijk MC, Tanis PJ, Nieweg OE, et al. Clinical implications of sentinel nodes outside the axilla and internal mammary chain in patients with breast cancer. J Surg Oncol. 2006;94:281-286.

31. Johnson MT, Guidroz JA, Smith BJ, et al. A single institutional experience of factors affecting successful identification of sentinel lymph node in breast cancer patients. Surgery. 2009;146:671-676.

32. Brenot-Rossi I, Houvenaeghel G, Jacquemier J, et al. Nonvisualization of axillary sentinel node during lymphoscintigraphy: is there a pathologic significance in breast cancer? J Nucl Med. 2003;44:1232-1237.

33. Lerman H, Lievshitz G, Zak O, Metser U, Schneebaum S, Even-Sapir E. Improved sentinel node identification by SPECT/CT in overweight patients with breast cancer. $J$ Nucl Med. 2007;48:201-206.

34. van der Ploeg IM, Nieweg OE, Kroon BB, et al. The yield of SPECT/CT for anatomical lymphatic mapping in patients with breast cancer. Eur J Nucl Med Mol Imaging. 2009;36:903-909.

35. Ibusuki M, Yamamoto Y, Kawasoe T, et al. Potential advantage of preoperative three-dimensional mapping of sentinel nodes in breast cancer by a hybrid single photon emission CT (SPECT)/CT system. Surg Oncol. 2010;19:88-94.

36. Bourgeois P, Nogaret JM, Veys I, et al. Isotope labelling and axillary node harvesting strategies for breast cancer. Eur J Surg Oncol. 2008;34:615-619.

37. Giard S, Chauvet MP, Penel N, et al. Feasibility of sentinel lymph node biopsy in multiple unilateral synchronous breast cancer: results of a French prospective multi-institutional study (IGASSU 0502). Ann Oncol. 2010;21:1630-1635.

38. Chung A, Giuliano A. Axillary staging in the neoadjuvant setting. Ann Surg Oncol. 2010;17:2401-2410.

39. Menard JP, Extra JM, Jacquemier J, et al. Sentinel lymphadenectomy for the staging of clinical axillary node-negative breast cancer before neoadjuvant chemotherapy. Eur J Surg Oncol. 2009;35:916-920.

40. Classe JM, Bordes V, Campion L, et al. Sentinel lymph node biopsy after neoadjuvant chemotherapy for advanced breast cancer: results of Ganglion Sentinelle et Chimiotherapie Neoadjuvante, a French prospective multicentric study. J Clin Oncol. 2009;27:726-732.

41. Baruah BP, Goyal A, Young P, Douglas-Jones AG, Mansel RE. Axillary node staging by ultrasonography and fine-needle aspiration cytology in patients with breast cancer. Br J Surg. 2010;97:680-683.

42. Heusner TA, Kuemmel S, Hahn S, et al. Diagnostic value of full-dose FDG PET/CT for axillary lymph node staging in breast cancer patients. Eur J Nucl Med Mol Imaging. 2009;36:1543-1550.

43. Groheux D, Moretti JL, Baillet G, et al. Effect of ${ }^{18}$ F-FDG PET/CT imaging in patients with clinical stage II and III breast cancer. Int J Radiat Oncol Biol Phys. 2008;71:695-704.

44. Groheux D, Giacchetti S, Espié M, Rubello D, Moretti JL, Hindié E. Early monitoring of response to neoadjuvant chemotherapy in breast cancer with ${ }^{18}$ F-FDG PET/CT: defining a clinical aim. Eur J Nucl Med Mol Imaging. November 12, 2010 [Epub ahead of print].

45. Julian TB, Blumencranz P, Deck K, et al. Novel intraoperative molecular test for sentinel lymph node metastases in patients with early-stage breast cancer. J Clin Oncol. 2008;26:3338-3345.
46. Chen RC, Lin NU, Golshan M, Harris JR, Bellon JR. Internal mammary nodes in breast cancer: diagnosis and implications for patient management-a systematic review. J Clin Oncol. 2008;26:4981-4989.

47. Estourgie SH, Tanis PJ, Nieweg OE, Valdés Olmos RA, Rutgers EJ, Kroon BB. Should the hunt for internal mammary chain sentinel nodes begin? An evaluation of 150 breast cancer patients. Ann Surg Oncol. 2003;10:935-941.

48. Paredes P, Vidal-Sicart S, Zanón G, et al. Clinical relevance of sentinel lymph nodes in the internal mammary chain in breast cancer patients. Eur J Nucl Med Mol Imaging. 2005;32:1283-1287.

49. Leidenius MH, Krogerus LA, Toivonen TS, Leppänen EA, von Smitten KA. The clinical value of parasternal sentinel node biopsy in breast cancer. Ann Surg Oncol. 2006;13:321-326.

50. Madsen E, Gobardhan P, Bongers V, et al. The impact on post-surgical treatment of sentinel lymph node biopsy of internal mammary lymph nodes in patients with breast cancer. Ann Surg Oncol. 2007;14:1486-1492.

51. Heuts EM, van der Ent FW, Hulsewé KW, von Meyenfeldt MF, Voogd AC. Results of tailored treatment for breast cancer patients with internal mammary lymph node metastases. Breast. 2009;18:254-258.

52. Bourre JC, Payan R, Collomb D, et al. Can the sentinel lymph node technique affect decisions to offer internal mammary chain irradiation? Eur J Nucl Med Mol Imaging. 2009;36:758-764.

53. van der Heiden-van der Loo M, Bezemer PD, Hennipman A, et al. Introduction of sentinel node biopsy and stage migration of breast cancer. Eur J Surg Oncol. 2006;32:710-714.

54. Hansen NM, Grube B, Ye X, et al. Impact of micrometastases in the sentinel node of patients with invasive breast cancer. J Clin Oncol. 2009;27:4679-4684.

55. Bilimoria KY, Bentrem DJ, Hansen NM, et al. Comparison of sentinel lymph node biopsy alone and completion axillary lymph node dissection for nodepositive breast cancer. J Clin Oncol. 2009;27:2946-2953.

56. Cox CE, Kiluk JV, Riker AI, et al. Significance of sentinel lymph node micrometastases in human breast cancer. J Am Coll Surg. 2008;206:261-268.

57. Tan LK, Giri D, Hummer AJ, et al. Occult axillary node metastases in breast cancer are prognostically significant: results in 368 node-negative patients with 20-year follow-up. J Clin Oncol. 2008;26:1803-1809.

58. de Boer M, van Deurzen CH, van Dijck JA, et al. Micrometastases or isolated tumor cells and the outcome of breast cancer. N Engl J Med. 2009;361:653-663.

59. Coutant C, Olivier C, Lambaudie E, et al. Comparison of models to predict nonsentinel lymph node status in breast cancer patients with metastatic sentinel lymph nodes: a prospective multicenter study. J Clin Oncol. 2009;27:2800-2808.

60. Houvenaeghel G, Nos C, Giard S, et al. A nomogram predictive of non-sentinel lymph node involvement in breast cancer patients with a sentinel lymph node micrometastasis. Eur J Surg Oncol. 2009;35:690-695.

61. Rodriguez Fernandez J, Martella S, Trifirò G, et al. Sentinel node biopsy in patients with previous breast aesthetic surgery. Ann Surg Oncol. 2009;16:989-992.

62. Gentilini O, Cremonesi M, Toesca A, et al. Sentinel lymph node biopsy in pregnant patients with breast cancer. Eur J Nucl Med Mol Imaging. 2010;37:78-83.

63. Virnig BA, Tuttle TM, Shamliyan T, Kane RL. Ductal carcinoma in situ of the breast: a systematic review of incidence, treatment, and outcomes. J Natl Cancer Inst. 2010;102:170-178.

64. Intra M, Rotmensz N, Veronesi P, et al. Sentinel node biopsy is not a standard procedure in ductal carcinoma in situ of the breast: the experience of the European institute of oncology on 854 patients in 10 years. Ann Surg. 2008;247:315-319.

65. Tunon-de-Lara C, Giard S, Buttarelli M, et al. Sentinel node procedure is warranted in ductal carcinoma in situ with high risk of occult invasive carcinoma and microinvasive carcinoma treated by mastectomy. Breast J. 2008;14:135-140.

66. Boneti C, Korourian S, Diaz Z, et al. Scientific Impact Award: axillary reverse mapping (ARM) to identify and protect lymphatics draining the arm during axillary lymphadenectomy. Am J Surg. 2009;198:482-487.

67. Bedrosian I, Babiera GV, Mittendorf EA, et al. A phase I study to assess the feasibility and oncologic safety of axillary reverse mapping in breast cancer patients. Cancer. 2010;116:2543-2548.

68. Mariscal Martínez A, Solà M, de Tudela AP, et al. Radioguided localization of nonpalpable breast cancer lesions: randomized comparison with wire localization in patients undergoing conservative surgery and sentinel node biopsy. AJR. 2009;193:1001-1009.

69. van Rijk MC, Tanis PJ, Nieweg OE, et al. Sentinel node biopsy and concomitant probe-guided tumor excision of nonpalpable breast cancer. Ann Surg Oncol. 2007;14:627-632.

70. Vermeeren L, Valdés Olmos RA, Klop WM, Balm AJ, van den Brekel MW. A portable gamma-camera for intraoperative detection of sentinel nodes in the head and neck region. J Nucl Med. 2010;51:700-703.

71. Canavese G, Catturich A, Vecchio C, et al. Sentinel node biopsy compared with complete axillary dissection for staging early breast cancer with clinically negative lymph nodes: results of randomized trial. Ann Oncol. 2009;20:1001-1007. 